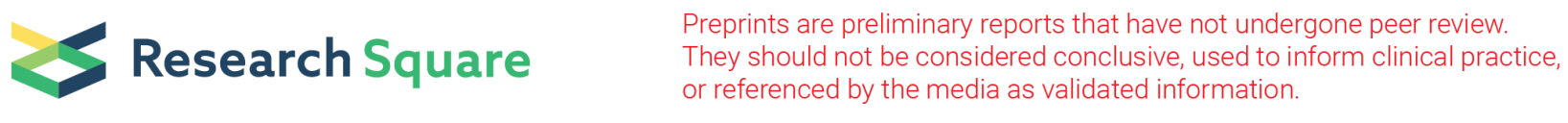

\title{
Early hemoglobin status is predictive of outcomes and long-term mortality of sepsis
}

\author{
Desheng Qi \\ Xiangya Hospital Central South University \\ Milin Peng ( $\nabla$ pengmilin@csu.edu.cn ) \\ Xiangya Hospital Central South University https://orcid.org/0000-0002-5443-5646
}

\section{Research}

Keywords: Sepsis, Hemoglobin, Erythrocyte, Critical Care, Mortality

Posted Date: February 11th, 2020

DOI: https://doi.org/10.21203/rs.2.23181/v1

License: (9) This work is licensed under a Creative Commons Attribution 4.0 International License. Read Full License 


\section{Abstract}

Background: Hemoglobin change plays a critical role in progress of sepsis. However, the contribution of hemoglobin change to outcomes of patients with sepsis is still unknown. The aim of our study is to investigate the effect of early hemoglobin level within 48h after admission to Intensive Care Unit (ICU) on long term prognosis for sepsis.

Methods: In this single centered, cohort study, we included patients from different ICU departments of Xiangya hospital from 2016 to 2018. Out of 1800 ICU patients, 199 patients with sepsis matched inclusion criteria. All 199 patients were divided into three groups according to 70,80 , and $90 \mathrm{~g} / \mathrm{L}$ hemoglobin statuses, respectively.

Results: Our study showed seventy-nine patients (39.7\%) with $\leq 90 \mathrm{~g} / \mathrm{L}$ hemoglobin, forty-seven patients $(23.6 \%)$ with hemoglobin $\leq 80 \mathrm{~g} / \mathrm{L}$, and twenty-five $(12.6 \%)$ with hemoglobin $\leq 70 \mathrm{~g} / \mathrm{L}$ at $48 \mathrm{~h}$ after admission. Compared to survival group, there were higher rates of patients with hemoglobin $\leq 80 \mathrm{~g} / \mathrm{L}(33.7 \%$ vs. $15.1 \%, P=0.003)$, hemoglobin $\leq 70 \mathrm{~g} / \mathrm{L}(18.5 \% \mathrm{vs} .7 .5 \%, P=0.031)$ in non-survival group, but similar rate of hemoglobin $\leq 90 \mathrm{~g} / \mathrm{L}$ patients $(46.7 \%$ vs. $34.0 \%, P=0.081)$. Kaplan-Meier survival analysis showed that significant difference occurred among all three groups with total one-year survival rates. Furthermore, multivariate cox proportional hazards regression analysis showed that serum hemoglobin concentration $\leq 80 \mathrm{~g} / \mathrm{L}$ at $48 \mathrm{~h}$ admission (Hazard Ratio HR 1.736, 95\% $\mathrm{Cl} 1.131-2.665, \mathrm{P}=0.012)$, the occurrence of ARDS (HR 1.814, 95\% Cl 1.184-2.778, $\mathrm{P}=0.006$ ), the use of CRRT (HR 1.569, 95\% Cl 1.0302.390, $\mathrm{P}=0.036$ ), and septic shock ( $\mathrm{HR} 1.776,95 \% \mathrm{Cl} 1.124-2.804, \mathrm{P}=0.014)$ were independent risk predictors for one-year mortality in septic patients. Whereas the infection site from abdomen ( $\mathrm{HR} 0.281,95 \% \mathrm{Cl} 0.113-0.697, \mathrm{P}=0.006)$ was the only independent protective factor for mortality in septic patients.

Conclusion: $\leq 80 \mathrm{~g} / \mathrm{L}$ hemoglobin within $48 \mathrm{~h}$ after admission to ICU was the independent risk factor for mortality for patients with sepsis. Attention should be raised up to target early hemoglobin level in course of sepsis to achieve better outcomes of sepsis. Future larger researches and randomized controlled trials are needed to validate our results.

\section{Background}

Hemoglobin change is commonplace, but emerges silently and easy to be ignored in critical care patients $(1,2)$. Sepsis is lifethreatening organ dysfunction caused by a dysregulated host immune and inflammatory response to infection and has high incidence rate and mortality. Sepsis is also the main cause for anemia in ICU $(3,4)$. Hemoglobin level continues to decline in the septic patients at the rate of $0.68 \pm 0.66 \mathrm{~g} / \mathrm{dL} /$ day but not in the nonseptic patients after the third day in ICU (5). The mechanism of hemoglobin variation in the course of sepsis is multifactorial covering inflammatory anemia, dilution of infusion, defective erythropoiesis, blunted bone marrow response to erythropoietin, preexiting conditions, hemolysis due to disseminated intravascular coagulation (DIC) or certain pathogens, adverse effect of drugs, repeated phlebotomy, nutrition deficit, et al $(6,7)$. Pathogens, host immune system, kidney, liver and bone marrow probably have complicated crosstalks contributing to the underlying anemia in sepsis. The leading risk associated with hemoglobin decrease is the shortage of oxygen-carrying capacity of blood resulting in tissue hypoxia. Afterwards hypoxia brings about extensive organ injury, which may be latent at the early stage but further aggravates in the late period of septic course. So maintaining early tissue oxygen supply is important to treat sepsis. Furthermore, hemoglobin plays a key role in defense against microorganism to assist leukocytes, nonspecific immunity as sequestering iron to cut off nutrition for bacteria, carring and transporting antibiotics, maintaining hemodynamic stabilization, carbon dioxide disposal, et al (8). So hemoglobin keeps internal environment homeostasis and the variation of hemoglobin is closely involved in the progress of sepsis. However, the hematologic system is often overlooked as an important organ in the treatment flowsheet of the patient with sepsis, and the impact of hemoglobin change on outcomes of patients with sepsis is presently unknown.

Several high quality randomized controlled trials have focused on transfusion threshold for making clinical decision for critical ill and sepsis patients $(9,10)$ and $70 \mathrm{~g} / \mathrm{L}$ level is recommended but still arousing dispute in clinical application. While recent meta-analysis showed no difference in 28- or 30-day mortality between the liberal (a hemoglobin threshold of 9 or $10 \mathrm{~g} / \mathrm{dL}$ ) and restrictive transfusion (a hemoglobin threshold of $7 \mathrm{~g} / \mathrm{dL}$ ) strategies in patients with sepsis (11). However, hemoglobin status is distinct from transfusion for disparate impact on septic outcomes (12). Whereas few studies have explored the effect of early hemoglobin level on long term outcome of sepsis patients in ICU. Previous study has shown relationship between hemoglobin level and clinical outcome of septic patients, but whose grouping strategy including hemoglobin $\geq 7-<10 \mathrm{~g} / \mathrm{dl}$ is so wide that probably missing detailed 
information of 8 or $9 \mathrm{~g} / \mathrm{dl}$ on prognosis (12). However, the optimal hemoglobin level for good prognosis of sepsis still remains unclear. Hence, we want to expore further to determine the best fittable early hemoglobin level for better outcomes of sepsis.

Therefore, in this single centered, cohort study, we evaluated the effect of early hemoglobin level of sepsis patients within 48 hours of ICU admission on long term prognosis, with the aim to investigate the most befitting early hemoglobin for better outcome of sepsis.

\section{Methods}

\section{Study design and population}

All patients who diagnosed with sepsis were admitted to ICU wards of Xiangya Hospital of Central South University for two years (January 1, 2016 to February 1, 2018). The study was conducted in accordance with STrengthening the Reporting of OBservational studies in Epidemiology (STROBE) statement. The data retrospectively collection was approved by the Ethics Committee of Xiangya Hospital. During the collection process, we adopted the basic information as hiding the patient's name and hospital number and implemented a unified de-identification to protect patient privacy. For the nature of the study, patients' informed consent was not necessary, and the Institutional Review Board waived the need for informed consent. The inclusion criteria were showed as follows: diagnosed with sepsis; $\geq 18$ years old; ICU admission more than $48 \mathrm{~h}$; with laboratory parameters within $48 \mathrm{~h}$. The exclusion criteria were contained with age $<18$ years, patients admitted with acute bleeding (gastrointestinal, trauma, postpartum hemorrhage, et al).

All patients underwent routine daily measure of serum level of hemoglobin. Patients with serum hemoglobin levels $>90 \mathrm{~g} / \mathrm{L}$ versus $\leq$ $90 \mathrm{~g} / \mathrm{L}$ at $48 \mathrm{~h}$ after admission were included in group 1, patients with serum hemoglobin levels $>80 \mathrm{~g} / \mathrm{L}$ versus $\leq 80 \mathrm{~g} / \mathrm{L}$ at $48 \mathrm{~h}$ after admission were included in group 2, and group 3 as serum hemoglobin levels $>70 \mathrm{~g} / \mathrm{L}$ versus $\leq 70 \mathrm{~g} / \mathrm{L}$ at $48 \mathrm{~h}$ after admission. We also investigated the clinical characteristics in sepsis by dividing to survival group and non-survival group according to survival state at hospital.

\section{Variable definition}

Sepsis was defined as life-threatening organ dysfunction caused by a dysregulated host response to infection according to Sepsis 3.0 criterion (infection plus an acute change in total Sequential Organ Failure Assessment, SOFA score $\geq 2$ points) (13). The definition of septic shock was sepsis with persisting hypotension requiring vasopressors to maintain mean arterial pressure (MAP) $\geq$ $65 \mathrm{mmHg}$ and having a serum lactate level $>2 \mathrm{mmol} / \mathrm{L}(18 \mathrm{mg} / \mathrm{dL})$ despite adequate volume resuscitation. Acute respiration distress syndrome (ARDS) is defined by Berlin criteria of 2012 (14), and the definition of AKI is according to adopted Kidney Disease: Improving Global Outcomes (KDIGO) clinical practice guideline (15).

\section{Data source}

We collected the clinical data from Electronic Hospital Records System (EHRs), including age, gender, co-morbid conditions, source of infection, vital signs at admission (heart rate, respiratory rate, and body temperature) and the clinical biochemical parameters at $48 \mathrm{~h}$ after admission to ICU (artery blood gas, blood routine, liver and kidney function, coagulation function, procalcitonin, myocardial enzymes, C-reaction protein, blood lipid full set), complications as septic shock, acute respiration syndrome (ARDS), and acute kidney injury (AKI) in septic patients, use of life support of the use of continues renal replication (CRRT), the use of vasoactive drug, mechanical ventilation (MV), the length of vasoactive drug usage days. All survival patients would be followed up by telephone at day 180 and 1-year after hospital discharge.

The primary outcome was defined as death from any cause within hospital, 180 days or one year after discharge. The secondary outcomes were defined as complications of sepsis (including septic shock, ARDS, AKI) and use of life support (the rate of CRRT, MV, vasopressor usage, days of using vasopressor, ICU hospitalization days, respectively).

\section{Statistical analysis}

Statistical analysis was performed using SPSS 23 (SPSS, Inc., Chicago, IL). Data were expressed as mean value (SD) for continuous variables, and as numbers (\%) for categorical variables. The mean values and frequencies were compared between two groups using unpaired student t-test and chi-square or Fisher exact test variables. The survival curves of those three groups according to the levels of hemoglobin were generated using the Kaplan-Meier survival analysis and the difference in survival among these groups was assessed using the log-rank test. Further, to adjust for confounding factors, the predictive values of mortality in sepsis were assessed 
by univariate and multivariate cox proportional hazards regression model, with hazard ratio (HR) and 95\% confidence interval (Cl). . In all comparisons, a $\mathrm{P}$ value of $\leq 0.05$ was considered statistically significant.

\section{Results}

From 1800 patients at ICU admission from January 1, 2016 to February 1, 2018, 199 patients were enrolled in the analysis (Fig. 1). 199 patients were included by screening through the inclusion the exclusion criteria in the Methods part. All 199 patients were divided by $>90 \mathrm{~g} / \mathrm{L}$ group $(n=120)$ and $\leq 90 \mathrm{~g} / \mathrm{L}$ group $(n=79),>80 \mathrm{~g} / \mathrm{L}$ group $(n=152)$ and $\leq 80 \mathrm{~g} / \mathrm{L}$ group $(n=47),>70 \mathrm{~g} / \mathrm{L}$ group $(n=$ $174)$ and $\leq 70 \mathrm{~g} / \mathrm{L}$ group $(n=25)$, respectively. For one-year follow-up, $110(84.2 \%)$ participants in $>90 \mathrm{~g} / \mathrm{L}$ group, $66(83.5 \%)$ in $\leq$ $90 \mathrm{~g} / \mathrm{L}$ group, 130 (85.5\%) in > $80 \mathrm{~g} / \mathrm{L}$ group, 37 (78.7\%) in $\leq 80 \mathrm{~g} / \mathrm{L}$ group, 148 (85.1\%) in > 70 g/L group, 19 (76\%) in $\leq 70 \mathrm{~g} / \mathrm{L}$ group were included in the longest follow-up of one year.

\section{Characteristics difference between survival and non-survival two groups}

Of the 199 patients, in-hospital mortality was shown in 92 (46.2\%) patients (Table 1). All 199 patients were grouped by in-hospital survival status. For general features and baseline, there are no significant difference in preexisting conditions between the two groups, but with significantly higher rate of male $(69.6 \%$ vs. $49.5 \%, P=0.006)$, higher SOFA score $(8.02 \pm 3.37$ vs. $5.33 \pm 3.02, P<$ $0.001)$ and lower rate of infection site from abdomen ( $5.4 \%$ vs. $15.9 \%, P=0.023)$ in non-survival group than survival group. The results showed higher septic shock rate $(81.5 \%$ vs. $35.5 \%, P<0.001)$, the occurrence of ARDS $(51.1 \%$ vs. $8.4 \%), P<0.001)$, the use of CRRT (39.1\% vs. $9.3 \%), P<0.001), M V(76.1 \%$ vs. $31.8 \%), P<0.001)$, and the frequency of vasopressors usage $(73.9 \%$ vs. $29.0 \%, P<$ $0.001)$, and longer days of using vasopressors (5.01 \pm 5.09 vs. $3.06 \pm 2.39, P=0.009)$ in non-survival group, compared to survival group. In different hemoglobin statuses, non-survival group had higher frequency of hemoglobin $\leq 80 \mathrm{~g} / \mathrm{L}$ patients $(33.7 \%$ vs. $15.1 \%$, $P=0.003)$, higher rate of hemoglobin $\leq 70 \mathrm{~g} / \mathrm{L}$ patients $(18.5 \% \mathrm{vs} .7 .5 \%, P=0.031)$, but similar rate of hemoglobin $\leq 90 \mathrm{~g} / \mathrm{L}$ patients (46.7\% vs. $34.0 \%, P=0.081)$, compared to survival group. Among other clinical parameters between these two groups, higher levels of lactic acid ( $3.26 \pm 3.15$ vs. $2.22 \pm 1.50, P=0.013$ ), activated partial thromboplastin time (APTT, $44.01 \pm 19.38$ vs. $38.20 \pm 9.91, P=$ $0.012)$ and $\mathrm{CK}-\mathrm{Mb}(55.58 \pm 112.88$ vs. $29.14 \pm 34.3, \mathrm{P}=0.036)$, lower levels of platelet $(120.60 \pm 106.09$ vs. $152.84 \pm 102.07, P=$ $0.030)$ and $\mathrm{pH}$ value $(7.38 \pm 0.91$ vs. $7.41 \pm 0.09, \mathrm{P}=0.049)$ were significantly shown in non-survival group, when compared with survival group. 
Table 1

Characteristics difference between survival and non-survival two groups according to survival status at hospital discharge.

\begin{tabular}{|c|c|c|c|}
\hline \multirow[t]{2}{*}{ Characteristics } & \multicolumn{2}{|l|}{ Full Cohort } & \multirow[t]{2}{*}{$\mathbf{P}$} \\
\hline & Non-survival group $(n=92)$ & Survival group $(n=107)$ & \\
\hline $\begin{array}{l}\text { General Features } \\
\text { Age, median (SD), }\end{array}$ & 60.63(16.91) & $57.65(15.18)$ & 0.192 \\
\hline Male, n (\%) & $64(69.6)$ & $53(49.5)$ & 0.006 \\
\hline \multicolumn{4}{|l|}{ Preexisting medical conditions, n (\%) } \\
\hline Connective tissue disease & $5(5.4)$ & $3(2.8)$ & 0.475 \\
\hline Gastrointestinal hemorrhage & $3(3.3)$ & $4(3.7)$ & 1.000 \\
\hline Coronary heart disease & 17(18.5) & 12(11.2) & 0.163 \\
\hline Hypertension & $25(27.2)$ & $29(27.1)$ & 1.000 \\
\hline SOFA at admission, median (SD) & $8.02(3.37)$ & $5.33(3.02)$ & $<0.001$ \\
\hline APACHEIl at admission, median (SD) & 23.30(18.81) & 17.81(19.23) & 0.125 \\
\hline \multicolumn{4}{|l|}{ Infection site, n (\%) } \\
\hline Urinary system & 16(18.6) & $20(18.9)$ & 1.000 \\
\hline Abdomen & $5(5.4)$ & 17(15.9) & 0.023 \\
\hline Respiratory tract & $59(64.1)$ & $58(54.2)$ & 0.194 \\
\hline \multicolumn{4}{|l|}{ Vital signs, median (SD) } \\
\hline Respiratory rate, median (SD) & $24.94(7.85)$ & 23.37(6.39) & 0.126 \\
\hline Body temperature, median (SD) & $37.57(2.92)$ & $37.26(0.90)$ & 0.296 \\
\hline $\begin{array}{l}\text { Different hemoglobin levels, } \mathrm{n}(\%) \\
\text { Hemoglobin }(\leq 90 \mathrm{~g} / \mathrm{L})\end{array}$ & $43(46.7)$ & $36(34.0)$ & 0.081 \\
\hline Hemoglobin $(\leq 80 \mathrm{~g} / \mathrm{L})$ & $31(33.7)$ & 16(15.1) & 0.003 \\
\hline Hemoglobin $(\leq 70 \mathrm{~g} / \mathrm{L})$ & 17(18.5) & $8(7.5)$ & 0.031 \\
\hline \multicolumn{4}{|l|}{ Other laboratory parameters } \\
\hline \multicolumn{4}{|l|}{ Artery blood gas, median (SD) } \\
\hline $\mathrm{pH}$ & 7.38(0.91) & $7.41(0.09)$ & 0.049 \\
\hline $\mathrm{pO}_{2}(>60 \mathrm{mmHg})$ & $68(80.0)$ & $71(84.5)$ & 0.547 \\
\hline $\mathrm{pCO}_{2}$ & $38.48(12.85)$ & $38.66(16.22)$ & 0.716 \\
\hline Oxygen concentration inhaled, $\mathrm{FiO} \%$ & $60.87(24.15)$ & $55.13(24.91)$ & 0.267 \\
\hline Oxygenation index & 178.54(109.57) & $224.00(135.62)$ & 0.077 \\
\hline
\end{tabular}

Continuous variables are reported as mean.

Abbreviations: SOFA sequential organ failure assessment, APACHE II Acute Physiology, Age, Chronic Health Evaluation II score, pCO2 partial pressure of carbon dioxide, pO2 partial pressure of oxygen, CRRT continuous renal replacement therapy, AKI acute kidney injury, ARDS acute respiratory distress syndrome, MV mechanical ventilation. WBC white blood cell, AST aspartate transaminase, ALT alanine transaminase, APTT activated partial thromboplastin time, PT prothrombin time, PTA prothrombin time activity percentage, GGT gamma-glutamyl transpeptidase, AKP alkline phosphatase, CK creatine kinase, CK-Mb creatine kinase isoenzyme, LDH lactate dehydrogenase, Mb myoglobin, TG triglyceride, CHOL cholesterol, LDL low density lipoprotein, HDL high density lipoprotein. 


\section{Non-survival group $(n=92) \quad$ Survival group $(n=107)$}

Lactic acid

Blood routine examination, median (SD)

Red blood cell

Hematocrit (\%)

WBC

Neutrophil (\%)

Neutrophil count

Lymphocyte (\%)

Lymphocyte count

Monocyte (\%)

Monocyte count

Platelet, median (SD)

Coagulation function test, median (SD)

APTT

PT

PTA

Procalcitonin, median (SD)

C-reaction protein, median (SD)

Blood lipid, median (SD)

TG

$\mathrm{CHOL}$

LDL

$\mathrm{HDL}$

Hepatic function, median (SD)

Albumin

ALT

AST

Total bilirubin

Direct bilirubin
3.26(3.15)

3.48(2.30)

$29.40(7.42)$

12.87(9.18)

81.94(16.98)

11.31(8.83)

10.43(12.47)

0.89(1.28)

5.62(4.90)

0.63(0.64)

120.60(106.09)
2.22(1.50)

0.013

\subsection{7(0.79)}

$30.78(7.61)$

0.961

0.199

$15.13(14.32)$

0.195

81.76(13.61)

12.29(10.16)

10.76(12.50)

1.02(0.70)

6.89(9.31)

0.92(1.48)

152.84(102.07)

38.20(9.91)

0.012

44.01(19.38)

17.10(10.82)

0.971

17.04(10.66)

84.29(65.70)

0.453

95.71(139.48)

29.020(50.39)

133.28(164.49)

59.49(5.83)

0.871

102.04(147.84)

0.215
0.472

0.854

0.389

0.244

0.085

0.030
2.20(2.47)

3.40(1.72)

4.36(18.67)

2.15(10.11)

27.60(6.21)

159.72(601.98)

292.67(1009.69)

2.20(2.477)

18.55(36.85)
2.22(2.70)

3.40(1.53)

2.15(1.28)

0.89(0.81)

31.03(21.07)

0.135

134.45(384.15)

0.721

168.45(478.61)

0.259

2.22(2.708)

0.063

21.50(29.85)
0.967

0.974

0.365

0.340

Continuous variables are reported as mean.

Abbreviations: SOFA sequential organ failure assessment, APACHE II Acute Physiology, Age, Chronic Health Evaluation II score, pCO2 partial pressure of carbon dioxide, p02 partial pressure of oxygen, CRRT continuous renal replacement therapy, AKI acute kidney injury, ARDS acute respiratory distress syndrome, MV mechanical ventilation. WBC white blood cell, AST aspartate transaminase, ALT alanine transaminase, APTT activated partial thromboplastin time, PT prothrombin time, PTA prothrombin time activity percentage, GGT gamma-glutamyl transpeptidase, AKP alkline phosphatase, CK creatine kinase, CK-Mb creatine kinase isoenzyme, LDH lactate dehydrogenase, Mb myoglobin, TG triglyceride, CHOL cholesterol, LDL low density lipoprotein, HDL high density lipoprotein. 


\begin{tabular}{|c|c|c|c|}
\hline \multirow[t]{2}{*}{ Characteristics } & \multicolumn{2}{|l|}{ Full Cohort } & \multirow[t]{2}{*}{$\mathbf{P}$} \\
\hline & Non-survival group $(n=92)$ & Survival group $(n=107)$ & \\
\hline GGT & $128.90(148.62)$ & 138.33(195.23) & 0.576 \\
\hline AKP & 178.37(159.15) & $385.06(490.08)$ & 0.220 \\
\hline Bile acid & 13.78(24.47) & 25.68(24.88) & 0.968 \\
\hline \multicolumn{4}{|l|}{ Myocardial enzymology, median (SD) } \\
\hline CK & 793.19(2803.97) & $485.79(1904.86)$ & 0.372 \\
\hline CK-Mb & $55.58(112.88)$ & $29.14(34.30)$ & 0.036 \\
\hline LDH & $951.08(2364.91)$ & $556.95(778.95)$ & 0.136 \\
\hline $\mathrm{Mb}$ & $315.97(820.38)$ & 169.37(208.05) & 0.104 \\
\hline Creatinine, median (SD) & 188.85(156.43) & 170.66(187.69) & 0.463 \\
\hline \multicolumn{4}{|l|}{ Use of life support } \\
\hline CRRT usage, n (\%) & $36(39.1)$ & 10(9.3) & $<0.001$ \\
\hline MV usage, n (\%) & $70(76.1)$ & $34(31.8)$ & $<0.001$ \\
\hline Vasopressor usage, n (\%) & 68(73.9) & $31(29.0)$ & $<0.001$ \\
\hline Days of using vasopressor, median (SD) & $5.01(5.09)$ & $3.06(2.39)$ & 0.009 \\
\hline ICU hospitalization days, median (SD) & $10.48(8.46)$ & 13.49(23.68) & 0.348 \\
\hline \multicolumn{4}{|l|}{ Complications, n (\%) } \\
\hline Septic Shock & $75(81.5)$ & $38(35.5)$ & $<0.001$ \\
\hline AKI & $43(46.7)$ & $39(36.4)$ & 0.151 \\
\hline ARDS & $47(51.1)$ & $9(8.4)$ & $<0.001$ \\
\hline
\end{tabular}

Continuous variables are reported as mean.

Abbreviations: SOFA sequential organ failure assessment, APACHE II Acute Physiology, Age, Chronic Health Evaluation II score, pCO2 partial pressure of carbon dioxide, p02 partial pressure of oxygen, CRRT continuous renal replacement therapy, AKI acute kidney injury, ARDS acute respiratory distress syndrome, MV mechanical ventilation. WBC white blood cell, AST aspartate transaminase, ALT alanine transaminase, APTT activated partial thromboplastin time, PT prothrombin time, PTA prothrombin time activity percentage, GGT gamma-glutamyl transpeptidase, AKP alkline phosphatase, CK creatine kinase, CK-Mb creatine kinase isoenzyme, LDH lactate dehydrogenase, Mb myoglobin, TG triglyceride, CHOL cholesterol, LDL low density lipoprotein, HDL high density lipoprotein.

\section{Characteristics and outcomes of septic patients according to different early hemoglobin levels}

Further, we explored characteristics and outcomes of septic patients based on different early hemoglobin statuses within $48 \mathrm{~h}$ after admission to ICU (Table 2). The results showed that the baseline was parallel except for the frequency of patients whose infection site from urinary system were higher in $\leq 90 \mathrm{~g} / \mathrm{L}$ hemoglobin group compared with $>90 \mathrm{~g} / \mathrm{L}$ group ( $30.3 \%$ vs. $11.2 \%, P=0.001)$, and also higher in $\leq 80 \mathrm{~g} / \mathrm{L}$ hemoglobin group compared to $>80 \mathrm{~g} / \mathrm{L}$ group ( $35.6 \%$ vs. $13.6 \%, P=0.002)$. The primary result showed significantly higher in-hospital ( $64.6 \%$ vs. $40.7 \%, P=0.002), 180-d(86.5 \%$ vs. $59.2 \%, P=0.002)$, one-year $(89.2 \%$ vs. $63.1 \%, P=0.002)$ mortality in $\leq 80 \mathrm{~g} / \mathrm{L}$ hemoglobin group compared to $>80 \mathrm{~g} / \mathrm{L}$ group. $\leq 70 \mathrm{~g} / \mathrm{L}$ hemoglobin group had higher in-hospital (68\% vs. $43.1 \%, P=0.03), 180-d(89.5 \%$ vs. $62.2 \%, P=0.02)$ mortality than $>70 \mathrm{~g} / \mathrm{L}$ group, whlie $\leq 90 \mathrm{~g} / \mathrm{L}$ hemoglobin group only had higher one-year mortality $(78.8 \%$ vs. $62.4 \%, P=0.027)$ compared with $>90 \mathrm{~g} / \mathrm{L}$ group. As for the secondary results, the rates of septic complications were similar. The use of life support results showed higher frequency of MV usage $(62 \% \mathrm{vs} .45 .8 \%, P=0.03)$ and vasopressor usage $(59.5 \%$ vs. $43.3 \%, P=0.03)$ in $\leq 90 \mathrm{~g} / \mathrm{L}$ hemoglobin group compared with $>90 \mathrm{~g} / \mathrm{L}$ group; higher rates of CRRT 
usage $(34 \%$ vs. $19.7 \%, P=0.049)$ and $M V$ usage $(66 \%$ vs. $48 \%, P=0.044)$ in $\leq 80 \mathrm{~g} / \mathrm{L}$ hemoglobin group versus $>80 \mathrm{~g} / \mathrm{L}$ group; and higher rates of CRRT usage ( $40 \%$ vs. $20.7 \%, P=0.042)$ in $\leq 70 \mathrm{~g} / \mathrm{L}$ hemoglobin group compared to $>70 \mathrm{~g} / \mathrm{L}$ group.

Table 2

Characteristics and outcomes of septic patients in three groups according to different early hemoglobin levels at $48 \mathrm{~h}$ admission (70, $80,90 \mathrm{~g} / \mathrm{L})$ in sepsis.

\begin{tabular}{|c|c|c|c|c|c|c|c|c|c|}
\hline \multirow{3}{*}{$\begin{array}{l}\text { Characteristics } \\
\text { and outcomes }\end{array}$} & \multicolumn{9}{|c|}{ The level of hemoglobin within $48 \mathrm{~h}$ on admission } \\
\hline & \multicolumn{3}{|l|}{ Group1 } & \multicolumn{3}{|l|}{ Group2 } & \multicolumn{3}{|l|}{ Group3 } \\
\hline & $\begin{array}{l}>90 \mathrm{~g} / \mathrm{L} \\
(\mathrm{n}=120)\end{array}$ & $\begin{array}{l}\leq 90 \mathrm{~g} / \mathrm{L} \\
(\mathrm{n}=79)\end{array}$ & $P$ & $\begin{array}{l}>80 \mathrm{~g} / \mathrm{L} \\
(\mathrm{n}=152)\end{array}$ & $\begin{array}{l}\leq 80 \mathrm{~g} / \mathrm{L} \\
(\mathrm{n}=47)\end{array}$ & $\mathrm{P}$ & $\begin{array}{l}>70 \mathrm{~g} / \mathrm{L} \\
(\mathrm{n}=174)\end{array}$ & $\begin{array}{l}\leq 70 \mathrm{~g} / \mathrm{L} \\
(\mathrm{n}=25)\end{array}$ & $P$ \\
\hline Age, M (SD) & $59.2(15.1)$ & $58.7(17.4)$ & 0.819 & $59.0(15.7)$ & 59.1(17.3) & 0.962 & $58.8(15.8)$ & $60.7(17.8)$ & 0.583 \\
\hline Male, n (\%) & $71(59.2)$ & $46(58.2)$ & 1.000 & $88(57.9)$ & $29(61.7)$ & 0.735 & $100(57.5)$ & $17(68.0)$ & 0.388 \\
\hline \multicolumn{10}{|c|}{ Infection site, $\mathrm{n}(\%)$} \\
\hline Urinary & 13(11.2) & $23(30.3)$ & 0.001 & $20(13.6)$ & 16(35.6) & 0.002 & $28(16.7)$ & $8(33.3)$ & 0.088 \\
\hline Abdomen & 16(13.3) & $6(7.6)$ & 0.252 & 20(13.2) & $2(4.3)$ & 0.112 & $20(11.5)$ & $2(8.0)$ & 1.000 \\
\hline Respiratory & $76(63.3)$ & $41(51.9)$ & 0.141 & $92(60.5)$ & $25(53.2)$ & 0.400 & $104(59.8)$ & $13(52.0)$ & 0.517 \\
\hline SOFA & $6.4(3.4)$ & $6.9(3.5)$ & 0.243 & $6.5(3.5)$ & $6.9(3.5)$ & 0.401 & $6.6(3.4)$ & $6.6(3.8)$ & 0.946 \\
\hline APACHEII & $11.8(19.5)$ & 13.2(15.8) & 0.595 & 12.2(19.7) & $12.9(11.7)$ & 0.816 & 12.7(19.1) & $9.6(8.4)$ & 0.415 \\
\hline \multicolumn{10}{|c|}{ Complications, n(\%) } \\
\hline Septic shock & $62(51.7)$ & $51(64.6)$ & 0.081 & $84(55.3)$ & $29(61.7)$ & 0.502 & $101(58.0)$ & $12(48.0)$ & 0.391 \\
\hline AKI & $49(40.8)$ & $33(41.8)$ & 1.000 & $60(39.5)$ & $22(46.8)$ & 0.400 & $67(38.5)$ & $15(60.0)$ & 0.051 \\
\hline ARDS & $33(27.5)$ & 23(29.1) & 0.872 & $41(27.0)$ & 15(31.9) & 0.578 & $47(27.0)$ & $9(36.0)$ & 0.350 \\
\hline \multicolumn{10}{|c|}{ Use of life support } \\
\hline CRRT, n(\%) & 23(19.2) & 23(29.1) & 0.123 & $30(19.7)$ & 16(34.0) & 0.049 & $36(20.7)$ & $10(40.0)$ & 0.042 \\
\hline $\mathrm{MV}, \mathrm{n}(\%)$ & $55(45.8)$ & $49(62.0)$ & 0.030 & $73(48.0)$ & $31(66.0)$ & 0.044 & $88(50.6)$ & $16(64.0)$ & 0.285 \\
\hline Vasopressor & $52(43.3)$ & $47(59.5)$ & 0.030 & $72(47.4)$ & $27(57.4)$ & 0.246 & $86(49.4)$ & $13(52.0)$ & 0.834 \\
\hline \multicolumn{10}{|l|}{ usage, $n(\%)$} \\
\hline \multicolumn{10}{|c|}{ Days of using vasopressor, median (SD) } \\
\hline & $2.2(3.9)$ & $2.5(3.8)$ & 0.617 & $2.2(3.7)$ & $2.6(4.5)$ & 0.495 & $2.2(3.7)$ & $3.1(5.4)$ & 0.286 \\
\hline \multicolumn{10}{|c|}{ ICU hospitalization days, median (SD) } \\
\hline & $9.5(20.2)$ & $9.0(8.9)$ & 0.868 & $9.5(18.6)$ & 8.7(9.3) & 0.788 & $9.5(17.7)$ & 7.7(8.7) & 0.636 \\
\hline \multicolumn{10}{|l|}{ Mortality, n(\%) } \\
\hline In-hospital & $49(40.8)$ & $43(54.4)$ & 0.081 & 61(40.7) & $31(64.6)$ & 0.002 & $75(43.1)$ & 17(68.0) & 0.030 \\
\hline $180-d$ & $60(59.4)$ & $49(74.2)$ & 0.067 & 77(59.2) & $32(86.5)$ & 0.002 & $92(62.2)$ & 17(89.5) & 0.020 \\
\hline 1-year & $63(62.4)$ & $52(78.8)$ & 0.027 & $82(63.1)$ & $33(89.2)$ & 0.002 & $98(66.2)$ & 17(89.5) & 0.062 \\
\hline \multicolumn{10}{|c|}{ Continuous variables are reported as mean. } \\
\hline $\begin{array}{l}\text { Abbreviations: } \\
\text { CRRT continuou } \\
\text { ventilation, ICU }\end{array}$ & $\begin{array}{l}\text { DFA sequenti } \\
\text { renal replac } \\
\text { itensive care }\end{array}$ & $\begin{array}{l}\text { I organ failu } \\
\text { ment therap } \\
\text { init. }\end{array}$ & $\begin{array}{l}\text { assessr } \\
\text { AKI acut }\end{array}$ & $\begin{array}{l}\text { nent, } A P A C H E ~ I I ~ A c \\
\text { e kidney injury, AR }\end{array}$ & $\begin{array}{l}\text { Ite Physiolo } \\
\text { S acute res }\end{array}$ & $\begin{array}{l}\text { Age, } \mathrm{Cl} \\
\text { atory di }\end{array}$ & $\begin{array}{l}\text { onic Health } \\
\text { tress syndro }\end{array}$ & $\begin{array}{l}\text { valuation II s } \\
\text { he, MV mech }\end{array}$ & $\begin{array}{l}\text { ore, } \\
\text { hical }\end{array}$ \\
\hline
\end{tabular}




\section{The survival curves}

The Kaplan-Meier survival analysis and log-rank test were used to access survival rates among the three groups (Fig. 2). There were significant differences among all three groups within total 1 year survival time, including the survival rate of $\leq 90 \mathrm{~g} / \mathrm{L}$ hemoglobin group significant lower than $>90 \mathrm{~g} / \mathrm{L}$ group $\left(X^{2}=7.696, \mathrm{P}=0.0055\right), \leq 80 \mathrm{~g} / \mathrm{L}$ hemoglobin group significant lower than $>80 \mathrm{~g} / \mathrm{L}$ group $\left(X^{2}=15.29, P<0.001\right)$, and $\leq 70 \mathrm{~g} / \mathrm{L}$ hemoglobin group significant lower than $>70 \mathrm{~g} / \mathrm{L}$ group $\left(X^{2}=11.06, P=0.0009\right)$.

\section{Univariate and multivariate Cox hazard analysis of risk factors for mortality in septic patients}

Further on, we used univariate and multiple Cox hazard analysis to investigate the independent risk factors of mortality in sepsis (Table 3). After backward elimination and controlling comfounders, at last, the rate of hemoglobin $\leq 90 \mathrm{~g} / \mathrm{L}$ at $48 \mathrm{~h}$ admission or the rate of hemoglobin $\leq 70 \mathrm{~g} / \mathrm{L}$ at $48 \mathrm{~h}$ admission were not entered into multiple Cox hazard model while hemoglobin $\leq 80 \mathrm{~g} / \mathrm{L}$ were incorporated. The results indicated that the rates of serum hemoglobin level $\leq 80 \mathrm{~g} / \mathrm{L}$ at $48 \mathrm{~h}$ admission (HR 1.736, 95\% Cl 1.1312.665, $\mathrm{P}=0.012)$, the occurrence of ARDS (HR 1.814, 95\% Cl 1.184-2.778, $\mathrm{P}=0.006)$, the use of CRRT (HR $1.569,95 \% \mathrm{Cl} 1.030-$ $2.390, P=0.036)$, and septic shock ( $H R 1.776,95 \% \mathrm{Cl} 1.124-2.804, P=0.014)$ were independent risk predictors for one-year mortality in septic patients. The frequency of infection site from abdomen (HR $0.281,95 \% \mathrm{Cl} 0.113-0.697, \mathrm{P}=0.006)$ was the only independent protective factor for one-year mortality in septic patients.

Table 3

Univariate and multivariate Cox hazard analysis of risk factors for mortality in patients with sepsis.

\begin{tabular}{|lllllll|}
\hline & \multicolumn{3}{c}{ Univariate analysis } & \multicolumn{3}{c|}{ Multivariate analysis } \\
\hline Factor & HR & $95 \% \mathrm{Cl}$ & $\mathrm{P}$ & HR & $95 \% \mathrm{Cl}$ & $\mathrm{P}$ \\
\hline $\begin{array}{l}\text { Hemoglobin } \leq 90 \mathrm{~g} / \mathrm{L} \\
\text { Hemoglobin } \leq 80 \mathrm{~g} / \mathrm{L}\end{array}$ & 1.621 & $1.119-2.347$ & 0.011 & - & $1.131-2.665$ & 0.012 \\
Hemoglobin $\leq 70 \mathrm{~g} / \mathrm{L}$ & 2.271 & $1.339-3.850$ & 0.002 & - & & \\
\hline ARDS & 2.647 & $1.800-3.892$ & $<0.001$ & 1.814 & $1.184-2.778$ & 0.006 \\
\hline MV & 2.632 & $1.764-3.926$ & $<0.001$ & 1.489 & $0.953-2.329$ & 0.081 \\
\hline CRRT & 2.438 & $1.630-3.647$ & $<0.001$ & 1.569 & $1.030-2.390$ & 0.036 \\
\hline Septic shock & 2.429 & $1.611-3.662$ & $<0.001$ & 1.776 & $1.124-2.804$ & 0.014 \\
\hline Infection site & & & & & & \\
\hline Abdomen & 0.318 & $0.130-0.781$ & 0.012 & 0.281 & $0.113-0.697$ & 0.006 \\
\hline Male & 1.532 & $1.031-2.276$ & 0.035 & 1.155 & $0.975-1.367$ & 0.095 \\
\hline
\end{tabular}

Abbreviations: CRRT continuous renal replacement therapy; ARDS acute respiratory distress syndrome. MV mechanical ventilation.

\section{Discussion}

In this cohort study, our results first show compared to $>80 \mathrm{~g} / \mathrm{L}$ group, septic patients with hemoglobin $\leq 80 \mathrm{~g} / \mathrm{L}$ within $48 \mathrm{~h}$ after admission to ICU has higher rates of in-hospital, 180-d and one-year mortality. The rates of CRRT and MV usage are also higher in $\leq$ $80 \mathrm{~g} / \mathrm{L}$ hemoglobin group. Furthermore, hemoglobin $\leq 80 \mathrm{~g} / \mathrm{L}$ within $48 \mathrm{~h}$ on admission,the occurrence of ARDS, the use of CRRT and 
septic shock are the independent risk factors of one-year mortality for septic patients. While the frequency of infectious source from abdomen was the only independent protective factor for one-year mortality in septic patients.

The comparison between survival and non-survival groups demonstrates the higher mortality rate for male septic patients, which is consistent with recent experimental studies $(16,17)$, sex-hormone-driven innate antibodies protect female from infection. And nonsurvival group has earlier hematologic and coagulation function turbulence, with higher rates of hemoglobin $\leq 80 \mathrm{~g} / \mathrm{L}$ and $\leq 70 \mathrm{~g} / \mathrm{L}$, lower platelet, longer APTT as shown by our data. So hematologic system plays a key role at the beginning of sepsis. Serum lactic acid level is higher in non-survival group partially represents tissue hypoxia in early stage. Higher rates of complications including septic shock and ARDS, higher frequency of use of life support, covering CRRT, MV and vasopressors usage, longer days of vasopressors and higher SOFA score in non-survival group reflect more serious of sepsis.

The nearly similar baseline precludes bias affecting results. Intriguingly, there are significantly higher in-hospital, 180-d, one-year mortality in $\leq 80 \mathrm{~g} / \mathrm{L}$ hemoglobin group compared to $>80 \mathrm{~g} / \mathrm{L}$ group in septic patients. While $\leq 90 \mathrm{~g} / \mathrm{L}$ hemoglobin group has the same in-hospital and 180-d mortality compared with $>90 \mathrm{~g} / \mathrm{L}$ group. These results hint at $80 \mathrm{~g} / \mathrm{L}$ hemoglobin could be the potential key cutoff to differentiate septic prognosis. However, there are significant differences of survival rates among all three hemoglobin groups within one year shown by Kaplan-Meier survival analysis.

Furthermore, we find $\leq 80 \mathrm{~g} / \mathrm{L}$ hemoglobin within $48 \mathrm{~h}$ after admission to ICU, the occurrence of ARDS, the use of CRRT, and septic shock are the independent risk factors of mortality of sepsis patients by multiple Cox hazard analysis to control possible cofounders. And the rate of infection site from abdomen is the only independent protective factor. Hemoglobin $\leq 90 \mathrm{~g} / \mathrm{L}$ is not the independent risk factor as not included in the multiple Cox hazard model. Hemoglobin $\leq 70 \mathrm{~g} / \mathrm{L}$ not incorporated into the model is possibly due to fewer cases. Albeit several large cohort shows $70 \mathrm{~g} / \mathrm{L}$ is the optimal transfusion threshold for critical patients including sepsis $(9,10)$, these results are not in contradiction with ours. Because we try to explore the best early hemoglobin level closely affecting sepsis prognosis, instead of blood transfusion threshold. We discuss the effective early hemoglobin level for delivering oxygen to important organs in early stage of sepsis, rather than the adverse reaction nor decreased oxygen utilization ability brought by transfusing stored-blood products (18). Our conclusion is in line with early goal-directed therapy (EGDT) study which emphasizes maintaining early tissue perfusion and correcting early tissue hypoxia are the core therapy strategy for sepsis (19). Time course of hemoglobin in nonbleeding ICU patients shows that hemoglobin of sepsis patients persists to decrease in whole course in ICU compared to nonseptic patients, and the change rate of hemoglobin concentration is inversely correlated to the severity of diseases (5). Several other studies results support our conclusion (20-23), while recent Sung' research has shown that $90 \mathrm{~g} / \mathrm{L}$ is the befitting cutoff hemoglobin level of 90-days mortality of patients with septic shock which is different from ours (24). However, there are two main differences between our study and Sung's: our study investigates much longer term, one year, prognosis of sepsis than Sung's, and our study subjects are septic patients while Sung's is the sick with septic shock. Patients with septic shock have more unstable hemodymic status thus probably requiring higher hemoglobin level to fulfill tissue oxygen need. Moreover, according our results hemoglobin $\leq$ $90 \mathrm{~g} / \mathrm{L}$ not influences in-hospital or 180-days mortality, secondly hemoglobin $\leq 90 \mathrm{~g} / \mathrm{L}$ is not independent risk factor of one-year mortality of septic patients in the process of using multivariate cox hazard model to control confounding bias. Studies with bigger data are needed to validate our results. In conclusion, we first report early hemoglobin level $>80 \mathrm{~g} / \mathrm{L}$ best fits better outcome and longer survival rate, this level is also recommended by experts consensus focused on hemodynamic support of sepsis and adopted by extensive clinicians, although lack of robust evidences (25).

In our results, the higher rates of septic shock and ARDS are independent risk factors for septic mortality is reasonable. However, whether the use of CRRT is beneficial or detrimental to septic patients is always in eternal debate. There are other factors that cannot be ignored should be taken into comprehensive consideration, that is, various pharmacokinetic changes of diverse antibiotics under different renal replacement therapies among distinct individuals in sepsis (26-28). So in order to maximize therapeutic effect of antibiotics and minimize its adverse effects, dynamic monitoring of individual pharmacokinetic change of specific antibiotic is needed when accessing effect of certain kind of renal replacement therapy on sepsis. As for infection site, one study shows abdominal infection site is the independent protective factor of in-hospital mortality of sepsis associated acute lung injury, which is consistent with our results (29). To verify the exact mechanism needs further experimental studies.

We suppose the underlying rationality for our conclusion is mainly associated with relationship between decreasing hemoglobin and early tissue and organ hypoxia at the beginning of sepsis. Hypoxia works in pathophysiology of abundant diseases including sepsis (30). We propose that hypoxia in early stage of sepsis plays an important role in the progress and bad outcome of sepsis, as the only 
carrier for oxygen in circulation, hemoglobin level in early phase of sepsis should be paid more attention. In addition, erythrocyte has many other important functions for defending against infection and sepsis except for oxygen carrier, for instance red blood cells can help kill bacteria more efficiently (31). Changes in properties of erythrocyte are also early sensitive warning signals for monitoring progress of sepsis $(32,33)$.

On the other hand, we want to emphasize that a simple hemoglobin cutoff should not be overemphasized against every unique scene in clinical practice. Because individual variation is universal between every two single persons, and different age, background co-morbidities, etiologies of sepsis, every respect should be taken into careful consideration for physicians to make decisions to cure patients. The latest editorial warns that the only hemoglobin concentration strength cannot be exaggerated instead we should take all the factors into account in every specific clinical scene (34), which is in line with our viewpoint. So we must stand beside the beds of patients, keep looking the specific dynamic change of conditions of every unique individual, weight the advantages and disadvantages for making transfusion and other decisions to achieve best clinical outcome for patients (35).

There are some limitations for our study. First, the type of our research is single centered, retrospective design and unavoidable for bias. We try our best to reduce confounding bias by using Cox proportional hazards regression model to prove the unique ability of early hemoglobin level predicting sepsis prognosis. What' more, we minimize cases that lose to follow-up and guaranteed follow-up equality to decrease withdraw bias; Secondly, our sample size is relatively not very big to enhance convince ability, while we complement the data to the greatest extent and do our best to decrease missing and guarantee data integrity and quality to strengthen stringency. Third, the single centered design restricts the generalizability of our conclusion, but we conducte the research in different ICUs setting to narrow generalizability reduction, multiple centered plan is suggested in the future. Fourth, $\leq 70$ hemoglobin not including in multivariate cox hazard model is due to the case number of $\leq 70$ hemoglobin was so small, future study with larger data is needed to verify our result.

There are some strengthens of our study. First, we focus on the influence of early hemoglobin level, grouped by $70,80 \mathrm{and} 90 \mathrm{~g} / \mathrm{L}$, on the long term prognosis of sepsis, which was not investigated before. And we found $\leq 80 \mathrm{~g} / \mathrm{L}$ hemoglobin is the independent risk factor for sepsis longer mortality, which is meaningful to raise awareness to value maintaining early hemoglobin stable in therapy for sepsis. Secondly, we suggest readers to interpret our results with discretion and call for uniting our results with actual situation of patient at the bedside to make best clinical decisions.

\section{Conclusion}

We suggest that $\leq 80 \mathrm{~g} / \mathrm{L}$ hemoglobin within $48 \mathrm{~h}$ on ICU admission is the independent predictor for longer term mortality for patients with sepsis, meanwhile we should also make decisions according to the characteristics of each patient in specific clinical situation. Furthermore, future studies with high quality of bigger data and randomized controlled trials are called for to validate our results and experimental researches are needed to illuminate underlying mechanism of the early tissue hypoxia and its compensation system in the progression of sepsis.

\section{Abbreviations}

ICU, intensive care units; EHRs, Electronic Hospital Records System; SOFA, sequential organ failure assessment; APACHE II, Acute Physiology, Age, Chronic Health Evaluation Il; KDIGO, Kidney Disease: Improving Global Outcomes; AKI, acute kidney injury; RR, respiratory rate; $\mathrm{MV}$, time mechanical ventilation time; $\mathrm{CRRT}$, continuous renal replacement therapy; MAP, mean arterial pressure; ARDS, acute respiration distress syndrome; $\mathrm{pCO} 2$, partial pressure of carbon dioxide; pO2, partial pressure of oxygen; ALT, Alanine transaminase; AST, Aspartate transaminase; TB, total bilirubin; DB, direct bilirubin; GGT, gamma-glutamyl transpeptidase; TBA, total bile acid; PCT, procalcitonin; Lac, lactic acid; CK, creatine kinase; CK-MB, creatine kinase-MB; LDH, lactate dehydrogenase; Mb, myoglobin; APTT, activated partial thromboplastin time; PT, prothrombin time; PTA, prothrombin time activity percentage.

\section{Declarations}

\section{Ethics approval and consent to participate}

The study was approved by the Institutional Review Board of Xiangya Hospital Central South University which abides by the guidance of the Declaration of Helsinki. This was a retrospective study, all data from electronic medical record and we adequately 
protected patients' privacy, so consent requirements were waived as a result. IRB number 2017121160.

\section{Consent for publication}

Not applicable.

\section{Availability of data and materials}

All data generated or analyzed during this study are included in this published article.

\section{Competing interests}

The authors declare that they have no competing interests.

\section{Funding}

Not applicable.

\section{Authors' contributions}

Milin Peng contributed to the conception and design of the research; Desheng Qi and Milin Peng contributed to the acquisition and analysis of the data; Milin Peng and Desheng Qi contributed to interpret of the results; and Desheng Qi drafted the manuscript. All authors critically revised the manuscript, agree to be fully accountable for ensuring the integrity and accuracy of the work, and read and approved the final manuscript.

\section{Acknowledgments}

We are grateful for professional Professor Liu WW for kind help for consultation of statistical problems.

\section{References}

1. Ayling OGS, Ibrahim GM, Alotaibi NM, Gooderham PA, Macdonald RL. Anemia After Aneurysmal Subarachnoid Hemorrhage Is Associated With Poor Outcome and Death. Stroke. 2018;49(8):1859-65.

2. Cherry-Bukowiec JR, Engoren M, Wiktor A, Raghavendran K, Napolitano LM. Hepcidin and Anemia in Surgical Critical Care: A Prospective Cohort Study. Crit Care Med. 2018;46(6):e567-e74.

3. Rhodes A, Evans LE, Alhazzani W, Levy MM, Antonelli M, Ferrer R, et al. Surviving Sepsis Campaign: International Guidelines for Management of Sepsis and Septic Shock: 2016. Intensive Care Med. 2017;43(3):304-77.

4. Abstracts of the 33rd International Symposium on Intensive Care and Emergency Medicine. Brussels, Belgium. March 19-22, 2013. Crit Care. 2013;17 Suppl 2:P1-545.

5. Nguyen BV, Bota DP, Melot C, Vincent JL. Time course of hemoglobin concentrations in nonbleeding intensive care unit patients. Crit Care Med. 2003;31(2):406-10.

6. Jiang $\mathrm{Y}$, Jiang FQ, Kong F, An MM, Jin BB, Cao D, et al. Inflammatory anemia-associated parameters are related to 28-day mortality in patients with sepsis admitted to the ICU: a preliminary observational study. Ann Intensive Care. 2019;9(1):67.

7. Claessens YE, Fontenay M, Pene F, Chiche JD, Guesnu M, Hababou C, et al. Erythropoiesis abnormalities contribute to early-onset anemia in patients with septic shock. Am J Respir Crit Care Med. 2006;174(1):51-7.

8. Aird WC. The hematologic system as a marker of organ dysfunction in sepsis. Mayo Clin Proc. 2003;78(7):869-81.

9. Hebert PC, Wells G, Blajchman MA, Marshall J, Martin C, Pagliarello G, et al. A multicenter, randomized, controlled clinical trial of transfusion requirements in critical care. Transfusion Requirements in Critical Care Investigators, Canadian Critical Care Trials Group. N Engl J Med. 1999;340(6):409-17.

10. Holst LB, Haase N, Wetterslev J, Wernerman J, Guttormsen AB, Karlsson S, et al. Lower versus higher hemoglobin threshold for transfusion in septic shock. N Engl J Med. 2014;371(15):1381-91.

11. Hirano Y, Miyoshi Y, Kondo Y, Okamoto K, Tanaka H. Liberal versus restrictive red blood cell transfusion strategy in sepsis or septic shock: a systematic review and meta-analysis of randomized trials. Crit Care. 2019;23(1):262. 
12. Muady GF, Bitterman H, Laor A, Vardi M, Urin V, Ghanem-Zoubi N. Hemoglobin levels and blood transfusion in patients with sepsis in Internal Medicine Departments. BMC Infect Dis. 2016;16(1):569.

13. Singer M, Deutschman CS, Seymour CW, Shankar-Hari M, Annane D, Bauer M, et al. The Third International Consensus Definitions for Sepsis and Septic Shock (Sepsis-3). JAMA. 2016;315(8):801-10.

14. Ferguson ND, Fan E, Camporota L, Antonelli M, Anzueto A, Beale R, et al. The Berlin definition of ARDS: an expanded rationale, justification, and supplementary material. Intensive Care Med. 2012;38(10):1573-82.

15. Kellum JA, Lameire N, Group KAGW. Diagnosis, evaluation, and management of acute kidney injury: a KDIGO summary (Part 1). Crit Care. 2013;17(1):204.

16. Zeng Z, Surewaard BGJ, Wong CHY, Guettler C, Petri B, Burkhard R, et al. Sex-hormone-driven innate antibodies protect females and infants against EPEC infection. Nat Immunol. 2018;19(10):1100-11.

17. Koch MA. Sex Bias in Sepsis. Cell Host Microbe. 2018;24(5):613-5.

18. Marik PE, Sibbald WJ. Effect of stored-blood transfusion on oxygen delivery in patients with sepsis. JAMA. 1993;269(23):3024-9.

19. Rivers E, Nguyen B, Havstad S, Ressler J, Muzzin A, Knoblich B, et al. Early goal-directed therapy in the treatment of severe sepsis and septic shock. N Engl J Med. 2001;345(19):1368-77.

20. Vincent JL, Sakr Y, Sprung C, Harboe S, Damas P, Sepsis Occurrence in Acutely III Patients I. Are blood transfusions associated with greater mortality rates? Results of the Sepsis Occurrence in Acutely III Patients study. Anesthesiology. 2008;108(1):31-9.

21. Park DW, Chun BC, Kwon SS, Yoon YK, Choi WS, Sohn JW, et al. Red blood cell transfusions are associated with lower mortality in patients with severe sepsis and septic shock: a propensity-matched analysis*. Crit Care Med. 2012;40(12):3140-5.

22. Sakr Y, Lobo S, Knuepfer S, Esser E, Bauer M, Settmacher U, et al. Anemia and blood transfusion in a surgical intensive care unit. Crit Care. 2010;14(3):R92.

23. Ter Avest E, de Jong M, Brummer I, Wietasch GJ, Ter Maaten JC. Outcome predictors of uncomplicated sepsis. Int J Emerg Med. 2013;6(1):9.

24. Jung SM, Kim YJ, Ryoo SM, Kim WY. Relationship between low hemoglobin levels and mortality in patients with septic shock. Acute Crit Care. 2019;34(2):141-7.

25. Hollenberg SM, Ahrens TS, Annane D, Astiz ME, Chalfin DB, Dasta JF, et al. Practice parameters for hemodynamic support of sepsis in adult patients: 2004 update. Crit Care Med. 2004;32(9):1928-48.

26. Roberts JA, Abdul-Aziz MH, Lipman J, Mouton JW, Vinks AA, Felton TW, et al. Individualised antibiotic dosing for patients who are critically ill: challenges and potential solutions. Lancet Infect Dis. 2014;14(6):498-509.

27. Wong WT, Choi G, Gomersall CD, Lipman J. To increase or decrease dosage of antimicrobials in septic patients during continuous renal replacement therapy: the eternal doubt. Curr Opin Pharmacol. 2015;24:68-78.

28. Beumier M, Casu GS, Hites M, Seyler L, Cotton F, Vincent JL, et al. beta-lactam antibiotic concentrations during continuous renal replacement therapy. Crit Care. 2014;18(3):R105.

29. Fujishima S, Gando S, Daizoh S, Kushimoto S, Ogura H, Mayumi T, et al. Infection site is predictive of outcome in acute lung injury associated with severe sepsis and septic shock. Respirology. 2016;21(5):898-904.

30. Taccone FS, Su F, De Deyne C, Abdellhai A, Pierrakos C, He X, et al. Sepsis is associated with altered cerebral microcirculation and tissue hypoxia in experimental peritonitis. Crit Care Med. 2014;42(2):e114-22.

31. Minasyan HA. Erythrocyte and leukocyte: two partners in bacteria killing. Int Rev Immunol. 2014;33(6):490-7.

32. Bateman RM, Sharpe MD, Singer M, Ellis CG. The Effect of Sepsis on the Erythrocyte. Int J Mol Sci. 2017;18(9).

33. Poschl JM, Leray C, Ruef P, Cazenave JP, Linderkamp O. Endotoxin binding to erythrocyte membrane and erythrocyte deformability in human sepsis and in vitro. Crit Care Med. 2003;31(3):924-8.

34. Vincent JL. Transfusion thresholds: the dangers of guidelines based on randomized controlled trials. Intensive Care Med. 2020.

35. Long B, Koyfman A. Red Blood Cell Transfusion in the Emergency Department. J Emerg Med. 2016;51(2):120-30.

\section{Figures}




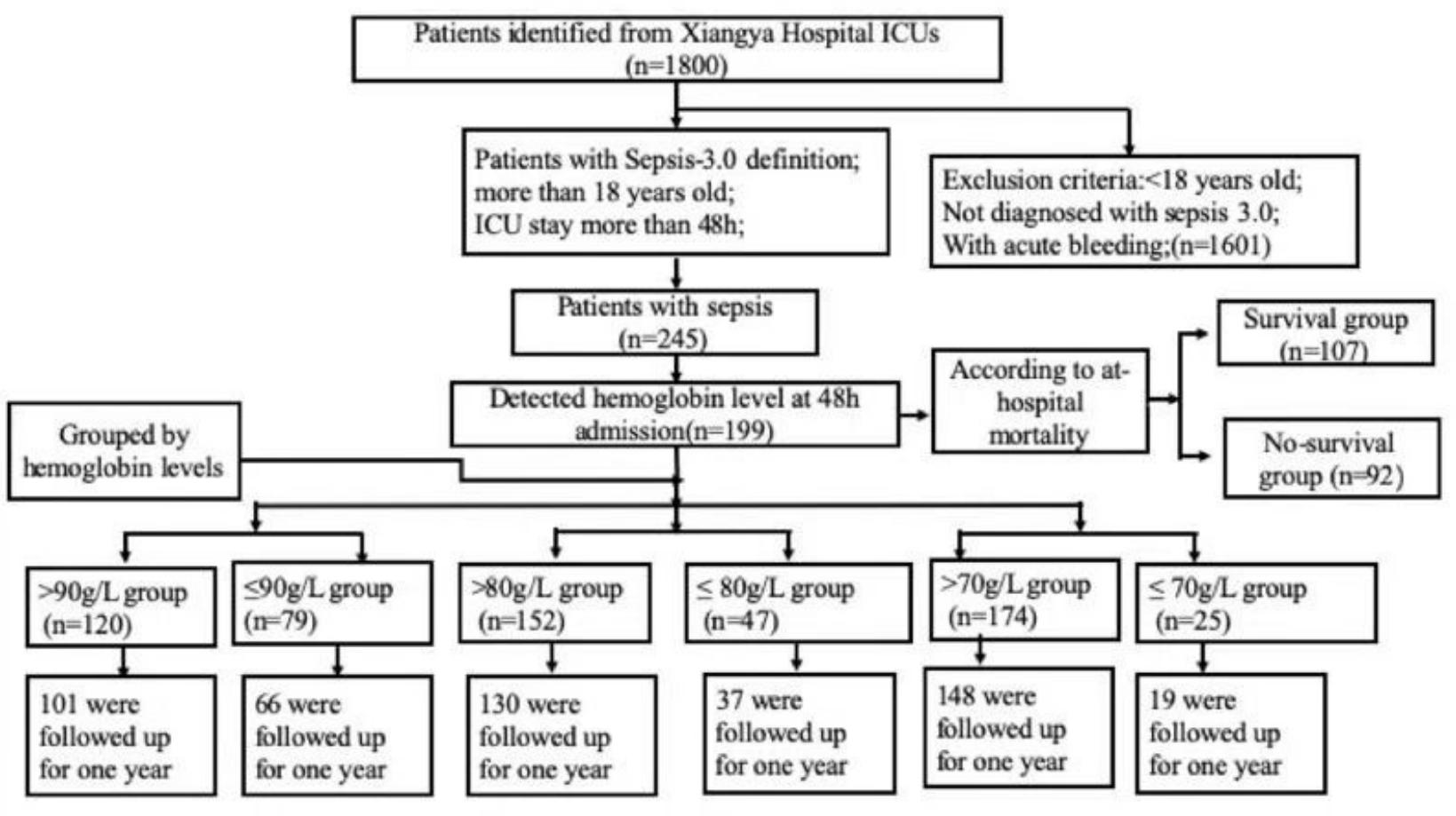

\section{Figure 1}

Flow chart for participants' number at each stage of study including follow-up. 
A

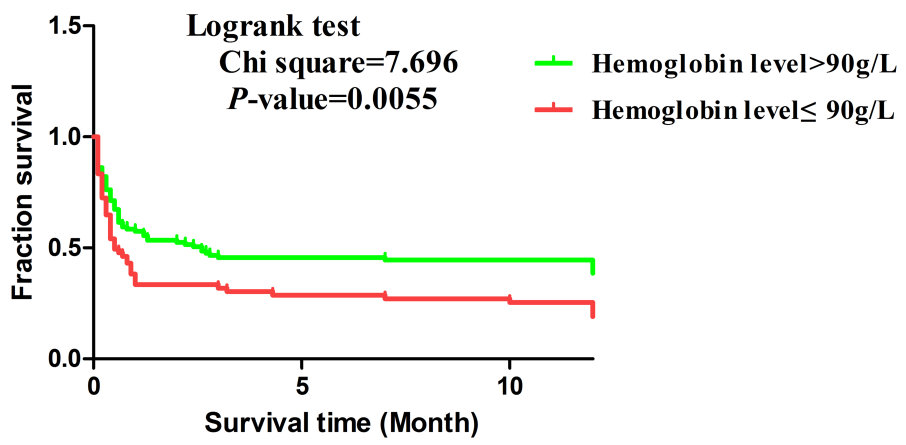

B

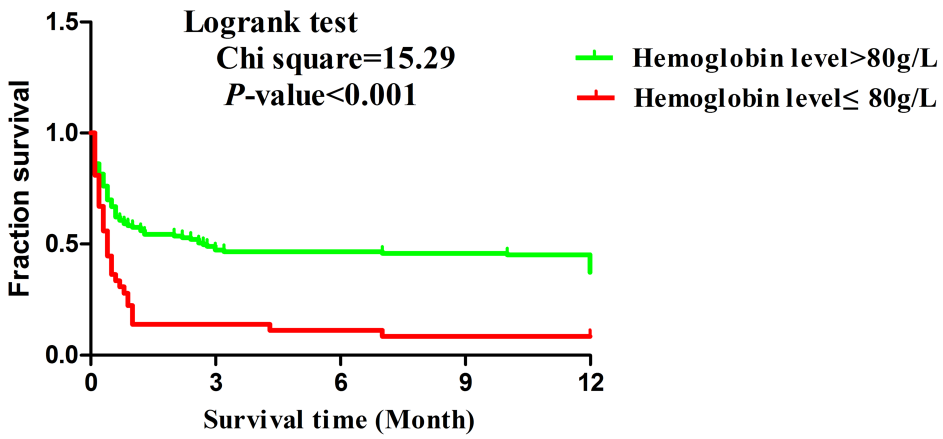

C

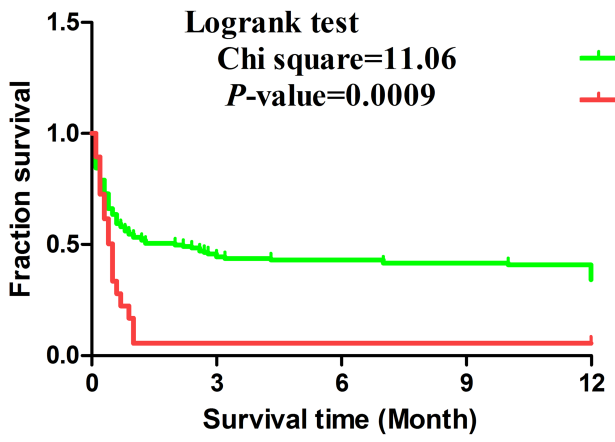

Figure 2

Time to death analysis. The figure shows the survival curves for all included patients at one year after discharge of the last patients according to levels of $70,80,90 \mathrm{~g} / \mathrm{L}$ hemoglobin statuses at $48 \mathrm{~h}$ admission in sepsis. There were significant differences among all three groups within total survival time of one year. A. The survival curves of group 1 (hemoglobin concentration at $48 \mathrm{~h}$ admission $>90 \mathrm{~g} / \mathrm{L}$ versus $\leq 90 \mathrm{~g} / \mathrm{L}$ group), B. The survival curves of group 2 (hemoglobin concentration at $48 \mathrm{~h}$ admission $>80 \mathrm{~g} / \mathrm{L}$ versus $\leq 80 \mathrm{~g} / \mathrm{L}$ group), and C. The survival curves of group 3 (hemoglobin concentration at $48 \mathrm{~h}$ admission $>70 \mathrm{~g} / \mathrm{L}$ versus $\leq 70 \mathrm{~g} / \mathrm{L}$ group). 